PHYSICAL REVIEW D 76, 025019 (2007)

\title{
Chiral anomaly beyond Lorentz invariance
}

\author{
Paola Arias* \\ Departamento de Física, Universidad de Santiago de Chile, Casilla 307, Santiago, Chile
}

H. Falomir ${ }^{\dagger}$

IFLP - Departamento de Física, Facultad de Ciencias Exactas, Universidad Nacional de la Plata, C.C. 67, (1900) La Plata, Argentina

\author{
J. Gamboa ${ }^{\ddagger}$ and F. Mendez ${ }^{\S}$ \\ Departamento de Física, Universidad de Santiago de Chile, Casilla 307, Santiago, Chile
}

\section{F. A. Schaposnik ${ }^{\|}$}

IFLP-Departamento de Física, Facultad de Ciencias Exactas, Universidad Nacional de la Plata, C.C. 67, (1900) La Plata, Argentina and CEFIMAS-SCA, Ave. Santa Fe 1145, C1059ABF, Buenos Aires, Argentina

(Received 28 May 2007; published 19 July 2007)

The chiral anomaly in the context of an extended standard model with Lorentz invariance violation is studied. Taking into account bounds from measurements of the speed of light, we argue that the chiral anomaly and its consequences are general results valid even beyond the relativistic symmetry.

DOI: 10.1103/PhysRevD.76.025019

PACS numbers: $12.38 . \mathrm{Aw}, 11.30 .-\mathrm{j}, 11.40 .-\mathrm{q}$

\section{INTRODUCTION}

Lorentz invariance is a cornerstone of relativistic quantum field theory (QFT) [1]. However, in the last years many authors have argued that at very high energy some symmetries, such as Lorentz and CPT, could be broken and, therefore, new scenarios and physical processes could take place [2-7].

Notice that Minkowski space and its isometries, the Lorentz transformations, should arise from a low energy solution of string theory. Therefore, it is a legitimate question to ask about a possible relic of this origin in a QFT at high energies.

However, even if a foremost invariance as the Lorentz one could be broken, one should expect that some important features and properties of quantum field theory are preserved, as well as the stability of some related phenomena.

In the context of a relativistic QFT with gauge fields and fermions, the chiral anomaly is related, as it is well known, to a topological object and is thus independent of the energy scale. This suggests that its form could be preserved even if Lorentz and $C P T$ symmetries were broken and, in particular, the $\pi^{0} \rightarrow 2 \gamma$ decay as derived from the chiral anomaly should be unaffected at any energy scale. So, it is worthwhile to consider this possibility in the framework of a quantum field theory model with an explicit Lorentz and/ or $C P T$ symmetry breaking.

\footnotetext{
*paola.arias@gmail.com

falomir@ fisica.unlp.edu.ar

¥jgamboa@usach.cl

\$fmendez@usach.cl

${ }_{\text {Associated with CICBA; }}$ fidel@ fisica.unlp.edu.ar
}

The purpose of this paper is to analyze the fate of the chiral anomaly in a model with an explicit Lorentz symmetry breaking and to explore, in connection with this, whether its role in connection with the $\pi^{0} \rightarrow 2 \gamma$ process is affected.

\section{A LORENTZ SYMMETRY VIOLATING MODEL FOR FERMIONS}

Let us start by considering the $d=4$ dimensional Lagrangian [8]

$$
\mathcal{L}=\bar{\psi}_{q} \Gamma^{\mu} D_{\mu}(A) \psi_{q},
$$

where $A_{\mu}=A_{\mu}^{a} T_{a}(\mu=0,1,2,3 ; a=1,2, \ldots, \operatorname{dim} \mathcal{G})$ are gauge fields taking values in the Lie algebra of some gauge group $G$ with generators $T_{a}$. Dirac fermions $\psi_{q}$ are taken in the fundamental representation of $G$ and $D_{\mu}$ is the usual covariant derivative. Concerning matrices $\Gamma^{\mu}$, they can in general take the form

$$
\Gamma^{\mu}=\gamma^{\mu}+\Gamma_{L V}^{\mu}+\Gamma_{C P T V}^{\mu} .
$$

Here $\gamma^{\mu}$ are the usual Dirac matrices while $\Gamma_{L V}^{\mu}$ and $\Gamma_{C P T V}^{\mu}$ are matrices which introduce violation of Lorentz and Lorentz-CPT symmetries, respectively. They are defined as

$$
\begin{gathered}
\Gamma_{L V}^{\mu}=c^{\mu}{ }_{\nu} \gamma^{\nu}+d^{\mu}{ }_{\nu} \gamma^{\nu} \gamma_{5}, \\
\Gamma_{C P T V}^{\mu}=e^{\mu}+f^{\mu} \gamma_{5}+g^{\mu \nu \lambda} \sigma_{\nu \lambda},
\end{gathered}
$$

where $c^{\mu}{ }_{\nu}, d^{\mu}{ }_{\nu}, e^{\mu}, f^{\mu}$, and $g^{\mu \nu \lambda}$ are real constants to be, in principle, phenomenologically determined.

But, if the theory is required to be invariant under (global) chiral transformations of the fermionic field at the classical level, then the $C P T$ violating terms must be removed since 


$$
\left\{\gamma_{5}, \Gamma_{C P T V}^{\mu}\right\} \neq 0 .
$$

So, we will take $e^{\mu}=0, f^{\mu}=0$, and $g^{\mu \nu \lambda}=0$.

On the other hand, although the $\Gamma^{\mu}$ matrices formally play the role of Dirac matrices, they do not satisfy in principle the standard Clifford algebra. Indeed, if we write

$$
\Gamma^{\mu}=\omega^{\mu}{ }_{\nu} \gamma^{\nu}+d^{\mu}{ }_{\nu} \gamma^{\nu} \gamma_{5} \text {, }
$$

where

$$
\omega_{\nu}^{\mu}=\delta_{\nu}^{\mu}+c_{\nu}^{\mu}
$$

it is straightforward to get

$$
\begin{aligned}
\left\{\Gamma^{\mu}, \Gamma^{\nu}\right\}= & \left(\omega^{\mu}{ }_{\alpha} \omega_{\beta}^{\nu}-d_{\alpha}^{\mu} d_{\beta}^{\nu}\right)\left\{\gamma^{\alpha}, \gamma^{\beta}\right\} \\
& +\left(\omega_{\alpha}^{\mu}{ }_{\alpha} d_{\beta}-d^{\mu}{ }_{\alpha} \omega^{\nu}{ }_{\beta}\right)\left[\gamma^{\alpha}, \gamma^{\beta}\right] \gamma_{5} .
\end{aligned}
$$

Then, if (in order to get a Clifford algebra for the $\Gamma$ matrices) we demand the last term in the right-hand side not to be present, we must impose that

$$
\left(\omega_{\alpha}^{\mu} d_{\beta}^{\nu}-d_{\alpha}^{\mu} \omega_{\beta}^{\nu}\right)-\left(\omega_{\beta}^{\mu} d_{\alpha}^{\nu}-d_{\beta}^{\mu} \omega_{\alpha}^{\nu}\right)=0 .
$$

Multiplying by $\left(\omega^{-1}\right)_{\mu}^{\alpha}$ (notice that $\omega^{\mu}{ }_{\alpha}$ is invertible, since we are looking for small Lorentz invariance violation (LIV)) we get

$$
4 d_{\beta}^{\nu}=\left[\left(\omega^{-1}\right)^{\alpha}{ }_{\mu} d_{\alpha}^{\mu}\right] \omega_{\beta}^{\nu},
$$

whose general solution is

$$
d_{\beta}^{\nu}=Q \omega_{\beta}^{\nu}
$$

with $Q$ a constant.

Consequently, we restrict our attention to this minimal Lorentz invariance violation, preserving chiral symmetry at the classical level and the form of the Clifford algebra, and take

$$
\Gamma^{\mu}=\omega_{\nu}^{\mu} \gamma^{\nu}\left(\mathbf{1}_{4}+Q \gamma_{5}\right) .
$$

Notice that

$$
\left\{\Gamma^{\mu}, \Gamma^{\nu}\right\}_{ \pm}=\left(1-Q^{2}\right) \omega_{\alpha}^{\mu} \omega_{\beta}^{\nu}\left\{\gamma^{\alpha}, \gamma^{\beta}\right\}_{ \pm},
$$

where we have taken $Q^{2} \ll 1$ since we shall consider small deviations from Lorentz invariance.

Therefore, the chosen set of $\Gamma$ matrices does fulfill the relations

$$
\begin{gathered}
\left\{\Gamma^{\mu}, \Gamma^{\nu}\right\}=2 M^{\mu \nu} \mathbf{1}_{4}, \\
\left\{\Gamma^{\mu}, \gamma_{5}\right\}=0,
\end{gathered}
$$

where $M^{\mu \nu}$ is a metric-like object defined as

$$
M^{\mu \nu}=\Omega_{\alpha}^{\mu} \Omega_{\beta}^{\nu} \eta^{\alpha \beta},
$$

where $\Omega^{\mu}{ }_{\alpha}=\omega^{\mu}{ }_{\alpha} \sqrt{1-Q^{2}}$ and $\eta^{\alpha \beta}$ is the standard metric in Minkowski space.

Notice that, since we are only interested in Lorentz violation effects in the fermion sector, we are also omitting a possible LIV term in the photon sector given by [9] $\left(\kappa_{F}\right)_{k \lambda \mu \nu} F^{k \lambda} F^{\mu \nu}$.

We shall present in the next section a derivation of the chiral anomaly for a quantum field theory in which the Fermi fields dynamics is governed by the Lorentz violating fermion Lagrangian in Eq. (1), testing whether the index theorem is still valid. We shall then see that neither the anomaly (Eq. (45)) nor the index theorem (Eq. (57)) are affected by the minimal Lorentz symmetry violation introduced in $\Gamma^{\mu}$. Only the axial current $\mathcal{J}_{5}^{\mu}$ is changed into

$$
\mathcal{J}_{5}^{\mu}=\omega^{\mu}{ }_{\nu}\left(j_{5}^{\nu}+Q j^{\nu}\right),
$$

where $j_{5}^{\nu}$ is the axial vector current arising in the ordinary Lorentz invariant case, $j_{5}^{\mu}=\bar{\psi}_{q} \gamma^{\mu} \gamma_{5} \psi_{q}$, and $j^{\mu}=$ $\bar{\psi}_{q} \gamma^{\mu} \psi_{q}$ is the vector current.

Before doing this, we shall discuss the situation from a more phenomenological point of view. To start up, let us assume that $c^{\mu}{ }_{\nu}$ has only one nonzero component, namely $c^{0}{ }_{0}=\kappa$. With this, rotational invariance is preserved and then

$$
\Gamma^{0}=\left(1+c_{0}^{0}\right) \gamma^{0}\left(\mathbf{1}_{4}+Q \gamma_{5}\right), \quad \Gamma^{i}=\gamma^{i}\left(\mathbf{1}_{4}+Q \gamma_{5}\right),
$$

and

$$
\left(M^{\mu \nu}\right)=\left(1-Q^{2}\right) \operatorname{diag}\left((1+\kappa)^{2},-1,-1,-1\right) .
$$

When replaced in the modified Dirac equation following from Lagrangian (1), this leads to the (free) dispersion relation

$$
\begin{aligned}
\Gamma^{\mu} \Gamma^{\nu} p_{\mu} p_{\nu} & =M^{\mu \nu} p_{\mu} p_{\nu}=\left(1-Q^{2}\right)\left\{(1+\kappa)^{2} p_{0}^{2}-\mathbf{p}^{2}\right\} \\
& =0,
\end{aligned}
$$

with $p_{0}=E / c$, where $c$ is the standard value of the velocity of light used here to set the length scale. Equation (19) implies that massless fermions $\psi_{q}$ move with velocity $v_{q}$ given by

$$
v_{q}=\frac{c}{1+\kappa}
$$

(with no dependence on the parameter $Q$ ).

Equation (20) of course implies new physics, in the vein of $[10,11]$ (see [12] for a complete list of references). Let us advance, however, that the results in the next section show that the minimal Lorentz invariance violation introduced here does not induce modifications in the form of the chiral anomaly. Nevertheless, it could imply modifications in the calculation of physical observables.

We shall now contrast our results with the experiment by considering, in a Lorentz violating invariance framework, the celebrated $\pi^{0} \rightarrow 2 \gamma$ process [13,14], directly related to chiral anomaly. In particular, let us analyze whether a departure from the usual chiral anomaly takes place by assuming that a formula similar to (20) (obtained for the case of massless fermions) also holds for photons. This can 
be justified as follows. In the present Lorentz invariance violating framework, one can relate the velocity of the photons with that of fermions in a given vertex through the formula

$$
c_{\mathrm{ph}}=n_{0} v_{q},
$$

where we have defined

$$
n_{0}=\frac{E_{\mathrm{ph}}}{E_{q}}
$$

with $E_{\mathrm{ph}}$ and $E_{q}$ the energies of photons and fermions, respectively.

Formula (21) can be obtained by cutting the standard triangle diagram (see Fig. 1) and using the 4-momentum conservation law for the process $\pi^{0} \rightarrow 2 \gamma$. Doing this one has

$$
\left(\frac{E_{q}}{v_{q}}, \vec{P}\right)+\left(\frac{E_{\bar{q}}}{v_{q}},-\vec{P}\right)=2\left(\frac{E_{\mathrm{ph}}}{c_{\mathrm{ph}}}, \overrightarrow{0}\right),
$$

and therefore

$$
\frac{E_{q}+E_{\bar{q}}}{v_{q}}=2 \frac{E_{\mathrm{ph}}}{c_{\mathrm{ph}}} .
$$

Since we are assuming that $C P T$ invariance is conserved, $E_{q}=E_{\bar{q}}$ and then Eq. (21) follows.

We shall consider the case in which the photon velocity $c_{\mathrm{ph}}$ does not exceed that of the neutral pion $v_{\pi^{0}}$ (in the case $c_{\mathrm{ph}}>v_{\pi^{0}}$ the decay is kinematically forbidden [11]). Also, interpreting $n_{0}$ in Eq. (21) as a refraction index, it is natural to take $n_{0} \approx 1$ in empty space. With all this, the off-massshell decay amplitude in the chiral limit is

$$
\begin{aligned}
\left(T\left(\pi^{0} \rightarrow 2 \gamma\right)_{\mathrm{LIV}}\right. & =\frac{\alpha^{2}}{64 \pi^{3}}\left(\frac{m_{\pi}}{f_{\pi}}\right)^{2} m_{\pi} c_{\mathrm{ph}}^{6} \\
& =\frac{\alpha^{2}}{64 \pi^{3}}\left(\frac{m_{\pi}}{f_{\pi}}\right)^{2} \frac{m_{\pi} c^{6}}{(1+\kappa)^{6}} .
\end{aligned}
$$

One can now estimate the ratio $T_{R} / T_{\mathrm{LIV}}$, with $T_{R}$ the relativistic rate of decay, using the bounds for $\kappa$ discussed in the literature. The comparison between the Lorentz violating invariance scheme and the relativistic one yields to
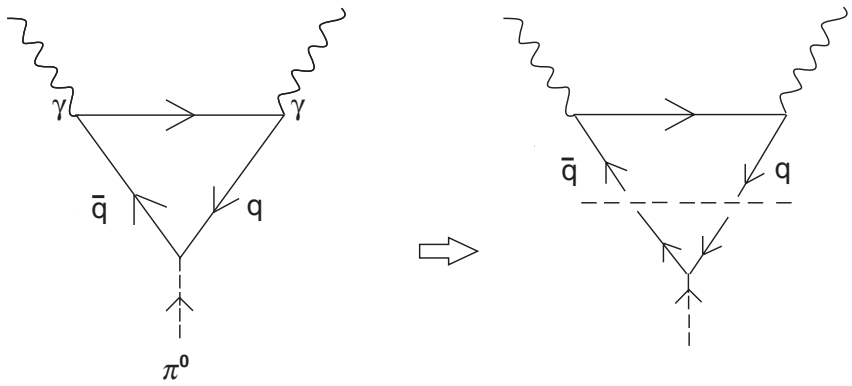

FIG. 1. Feynman diagram for pion decay.
TABLE I. Bounds on $\delta c$ given by different Lorentz invariance violation tests.

\begin{tabular}{lcc}
\hline \hline LIV tests & $\delta c / c \sim$ & Ref. \\
\hline Astrophysics & $10^{-19}$ & {$[15]$} \\
Atomic physics & $10^{-23}$ & {$[16]$} \\
Laser interferometry-anisotropy & $10^{-16}$ & {$[17]$} \\
Neutrino sector & $10^{-19}$ & {$[18]$} \\
GZK cutoff (theoretical) & $10^{-20}$ & {$[7]$} \\
Muon collider (theoretical) & $10^{-21}$ & {$[11]$} \\
Photon stability (theoretical) & $10^{-15}$ & {$[11]$} \\
\hline \hline
\end{tabular}

$$
\frac{(T)_{R}}{(T)_{\mathrm{LIV}}}-1 \approx 6 \kappa .
$$

In order to estimate the right-hand side in (26) let us introduce $\delta c$ through the equation

$$
\delta c=c-c_{\mathrm{ph}},
$$

where $c$ is the standard value of light velocity. Different experimental and phenomenological tests show the smallness of bounds on $\delta c$ (see Table I). Using (20) and (21) we can write

$$
\delta c=c-\frac{n_{0} c}{1+\kappa}
$$

or

$$
\kappa=\frac{\delta c}{c-\delta c}=\frac{\delta c}{c}+\mathrm{O}\left(\frac{\delta c^{2}}{c^{2}}\right),
$$

where we have again used $n_{0} \approx 1$. Bounds on $\delta c / c$ can then be translated into bounds on $\kappa$.

We see that the smallness of the bounds implies, at very high energy, that the chiral anomaly effects are effectively the same as in the relativistic invariant case. Indeed, one has that $(T)_{\mathrm{LIV}} /(T)_{R}-1$ takes values between $10^{-9}-10^{-22}$ and, therefore, Lorentz invariance deviations are experimentally almost unattainable.

Therefore, the changes in the decay $\pi^{0} \rightarrow 2 \gamma$ are extremely small, which is consistent with the universal character of the chiral anomaly.

One should note, however, that this conclusion could change drastically if the other terms not considered in our analysis are included. However, if we invoke the universal character of the anomaly then our assumption (11) is reasonably justified.

\section{THE CHIRAL ANOMALY AND THE INDEX THEOREM}

Let us consider a Hermitian Dirac operator that includes a minimal Lorentz symmetry violation as discussed in the previous section,

$$
D_{m}=D+m, \quad \text { with } \quad D=\Gamma^{\mu} D_{\mu},
$$


where

$$
D_{\mu}=i \frac{\partial}{\partial x^{\mu}}+A_{\mu}
$$

is the usual covariant derivative and the matrices $\Gamma^{\mu}$ are given in Eqs. (6) and (11). A small mass $m$ has been introduced in order to deal with zero modes. At the end of the calculation one should take the $m \rightarrow 0$ limit in a consistent way [19].

The $\Gamma$ matrices, depending on the constant tensor $c_{\nu}^{\mu}$ and the parameter $Q$ which breaks Lorentz symmetry, satisfy the Clifford algebra in Eq. (13) and anticommute with $\gamma_{5}$ as in Eq. (14). Their (anti)commutator is given in Eq. (12).

In order to analyze the issue of chiral symmetry we follow the Noether method starting from a $U(1)$ local chiral transformation,

$$
\psi(x) \rightarrow e^{i \alpha(x) \gamma_{5}} \psi(x), \quad \bar{\psi}(x) \rightarrow \bar{\psi}(x) e^{i \alpha(x) \gamma_{5}}
$$

The fermionic Lagrangian changes as

$$
\begin{aligned}
\mathcal{L} & =\bar{\psi}(x) D_{m} \psi(x) \rightarrow \bar{\psi}(x) e^{i \alpha(x) \gamma_{5}} D_{m} e^{i \alpha(x) \gamma_{5}} \psi(x) \\
& =\mathcal{L}+\bar{\psi}(x)\left\{i \alpha(x) \gamma_{5}, D_{m}\right\} \psi(x)+O\left(\alpha^{2}\right) .
\end{aligned}
$$

The first order in $\alpha$ on the right-hand side reduces, up to a total divergence, to

$$
\begin{aligned}
\delta \mathcal{L} & =\bar{\psi}(x)\left\{i \alpha(x) \gamma_{5}, D_{m}\right\} \psi(x) \\
& =\alpha(x)\left(\partial_{\mu} \mathcal{J}_{5}^{\mu}(x)+2 i m \bar{\psi}(x) \gamma_{5} \psi(x)\right),
\end{aligned}
$$

where the axial current is now given in Eq. (16).

Let us now consider the functional integral

$$
\begin{aligned}
Z & =\int \mathcal{D} \bar{\psi} \mathcal{D} \psi e^{-\int d^{4} x \mathcal{L}} \\
& =J[\alpha] \int \mathcal{D} \bar{\psi} \mathcal{D} \psi e^{-\int d^{4} x \mathcal{L}}\left(1+\int d^{4} x \delta \mathcal{L}+O\left(\alpha^{2}\right)\right)
\end{aligned}
$$

where

$$
\begin{aligned}
J[\alpha]= & \frac{\operatorname{det}\left(e^{i \alpha(x) \gamma_{5}} D_{m} e^{i \alpha(x) \gamma_{5}}\right)}{\operatorname{det} D_{m}} \\
= & 1-\int d^{4} x \alpha(x)\left\langle\partial_{\mu} \mathcal{J}_{5}^{\mu}(x)\right\rangle-2 i m \int d^{4} x \alpha(x) \\
& \times\left\langle\bar{\psi}(x) \gamma_{5} \psi(x)\right\rangle+O\left(\alpha^{2}\right)
\end{aligned}
$$

is the Jacobian [20,21] arising from the change in the fermionic measure under rotation (32).

Being the Jacobian ill-defined (the Dirac operator eigenvalues grow with no bound), one should introduce an appropriate regularization $(R)$. We use a heat-kernel regularization so that, up to $O\left(\alpha^{2}\right)$ terms, we have

$$
\begin{aligned}
\log J[\alpha]= & \left.\left(\operatorname{Tr} \log \left(D_{m}+\left\{i \alpha(x) \gamma_{5}, D_{m}\right\}\right)-\operatorname{Tr} \log D_{m}\right)\right|_{R} \\
= & \left.2 i \operatorname{Tr}\left(\alpha(x) \gamma_{5}\right)\right|_{R} \\
= & \left.2 i \lim _{\Lambda \rightarrow \infty} \operatorname{Tr}\left(\alpha(x) \gamma_{5} e^{-\left(D_{m}^{2} / \Lambda^{2}\right)}\right)\right|_{R} \\
= & 2 i \lim _{\Lambda \rightarrow \infty} \int d^{4} x \int \frac{d^{4} k}{(2 \pi)^{4}} \operatorname{tr}\left(\alpha(x) \gamma_{5}\right. \\
& \left.\times e^{-i k \cdot x} e^{-\left(D_{m}^{2} / \Lambda^{2}\right)} e^{i k \cdot x}\right),
\end{aligned}
$$

where

$$
D_{m}^{2}=\mathbf{1}_{4} M^{\mu \nu} D_{\mu} D_{\nu}+\frac{i}{4}\left[\Gamma^{\mu}, \Gamma^{\nu}\right] F_{\mu \nu}+O(m) .
$$

A straightforward calculation taking into account that

$$
\operatorname{tr}\left\{\gamma_{5}\left[\Gamma^{\mu}, \Gamma^{\nu}\right]\right\}=\Omega^{\mu}{ }_{\alpha} \Omega^{\nu}{ }_{\beta} \operatorname{tr}\left\{\gamma_{5}\left[\gamma^{\alpha}, \gamma^{\beta}\right]\right\}=0
$$

and $\operatorname{tr}\left\{\gamma_{5}\right\}=0$ leads to

$$
\begin{aligned}
\log J[\alpha]= & -\frac{i}{16} \int d^{4} x \int \frac{d^{4} k}{(2 \pi)^{4}} e^{-M^{\mu \nu} k_{\mu} k_{\nu}} \\
& \times \operatorname{tr}\left(\alpha(x) \gamma_{5}\left[\Gamma^{\mu}, \Gamma^{\nu}\right]\left[\Gamma^{\alpha}, \Gamma^{\beta}\right] F_{\mu \nu} F_{\alpha \beta}\right) \\
= & -\frac{i}{(16 \pi)^{2}}(\operatorname{det} M)^{-1 / 2} \Omega^{\mu}{ }_{\rho} \Omega^{\nu}{ }_{\sigma} \Omega^{\alpha}{ }_{\kappa} \Omega^{\beta}{ }_{\Omega} \\
& \times \int d^{4} x \operatorname{tr}\left(\alpha(x) \gamma_{5}\left[\gamma^{\rho}, \gamma^{\sigma}\right]\left[\gamma^{\kappa}, \gamma^{\Omega}\right] F_{\mu \nu} F_{\alpha \beta}\right),
\end{aligned}
$$

up to $O(m)$ terms.

Using

$$
\operatorname{tr}\left\{\gamma_{5} \gamma^{\rho} \gamma^{\sigma} \gamma^{\kappa} \gamma^{\Omega}\right\}=4 \epsilon^{\rho \sigma \kappa \Omega}
$$

one gets

$$
\begin{aligned}
\log J[\alpha]= & -\frac{i}{16 \pi^{2}} \int d^{4} x \operatorname{tr}\left(\alpha(x) \epsilon^{\rho \sigma \kappa \Omega} F_{\mu \nu} F_{\alpha \beta}\right) \\
& \times(\operatorname{det} M)^{-1 / 2} \Omega^{\mu}{ }_{\rho} \Omega^{\nu}{ }_{\sigma} \Omega^{\alpha}{ }_{\kappa} \Omega^{\beta}{ }_{\Omega} \\
= & -\frac{i}{16 \pi^{2}} \int d^{4} x \operatorname{tr}\left(\alpha(x) \epsilon^{\mu \nu \alpha \beta} F_{\mu \nu} F_{\alpha \beta}\right) \\
& \times(\operatorname{det} M)^{-1 / 2} \operatorname{det}(\Omega) .
\end{aligned}
$$

Finally, taking into account Eq. (15) one can see that all dependence on $\Omega_{\sigma}^{\rho}$ cancels out in the Jacobian,

$$
\log J[\alpha]=-\frac{i}{16 \pi^{2}} \int d^{4} x \alpha(x) F_{\mu \nu}^{a} F_{\alpha \beta}^{b} \epsilon^{\mu \nu \alpha \beta} \operatorname{tr}\left(T_{a} T_{b}\right)
$$

so that, after use of Eq. (36), one can write

$$
\begin{aligned}
& \frac{i}{16 \pi^{2}} \operatorname{tr} \int d^{4} x \alpha(x) F_{\mu \nu} F_{\alpha \beta} \epsilon^{\mu \nu \alpha \beta} \\
& =\int d^{4} x \alpha(x)\left\langle\partial_{\mu} \mathcal{J}_{5}^{\mu}(x)\right\rangle+\lim _{m \rightarrow 0} 2 \operatorname{Im} \int d^{4} x \alpha(x) \\
& \quad \times\left\langle\bar{\psi}(x) \gamma_{5} \psi(x)\right\rangle .
\end{aligned}
$$


Differentiating with respect to $\alpha$ one obtains the $U(1)$ anomaly equation in the form

$$
\begin{gathered}
\left\langle\partial_{\mu} \mathcal{J}_{5}^{\mu}(x)\right\rangle+\lim _{m \rightarrow 0} 2 \operatorname{Im}\left\langle\bar{\psi}(x) \gamma_{5} \psi(x)\right\rangle \\
=\frac{i}{16 \pi^{2}} \operatorname{tr}\left(F_{\mu \nu}(x) F_{\alpha \beta}(x)\right) \epsilon^{\mu \nu \alpha \beta} .
\end{gathered}
$$

Notice that the right-hand side of this equation is insensitive to the Lorentz symmetry breaking introduced by the tensor $c^{\mu}{ }_{\nu}$ and the parameter $Q$.

Let us now consider the contribution of the zero modes. The mean value of $\bar{\psi}(x) \gamma_{5} \psi(x)$ is given by

$$
\begin{aligned}
\left\langle\bar{\psi}(x) \gamma_{5} \psi(x)\right\rangle= & \frac{1}{Z} \int \mathcal{D} \bar{\psi} \mathcal{D} \psi \\
& \times e^{-\int \bar{\psi}(x) D_{m} \psi(x) d^{4} x} \bar{\psi}(x) \gamma_{5} \psi(x) .
\end{aligned}
$$

Let $\varphi_{n}$ be the eigenvectors of $D$,

$$
D \varphi_{n}=\Omega_{n} \varphi_{n} \Rightarrow D_{m} \varphi_{n}=\left(\Omega_{n}+m\right) \varphi_{n},
$$

with

$$
\int \varphi_{n}(x)^{\dagger} \varphi_{m}(x) d^{4} x=\delta_{n, m} .
$$

Some of them can be zero modes of $D$. Since $\left\{\gamma_{5}, \Gamma^{\mu}\right\}=0$, one can always choose these zero modes with a definite chirality,

$$
D \varphi_{0, k}^{ \pm}=0 \Rightarrow D_{m} \varphi_{0, k}^{ \pm}=m \varphi_{0, k}^{ \pm}, \quad k=1,2, \ldots, n_{ \pm},
$$

with

$$
\gamma_{5} \varphi_{0, k}^{ \pm}= \pm \varphi_{0, k}^{ \pm} .
$$

The integration variables in the functional integral can be expanded as

$$
\bar{\psi}=\sum_{n} \bar{c}_{n} \varphi_{n}^{\dagger}, \quad \psi=\sum_{n} c_{n} \varphi_{n},
$$

and the integration measure be written as

$$
\mathcal{D} \bar{\psi} \mathcal{D} \psi=\prod_{n} d \bar{c}_{n} d c_{n} .
$$

Therefore,

$$
\begin{aligned}
\left\langle\bar{\psi}(x) \gamma_{5} \psi(x)\right\rangle= & \frac{1}{Z} \int \prod_{n} d \bar{c}_{n} d c_{n} e^{-\sum_{n}\left(\Omega_{n}+m\right) \bar{c}_{n} c_{n}} \\
& \times \sum_{p, q} \bar{c}_{p} \varphi_{p}(x)^{\dagger} \gamma_{5} \varphi_{q}(x) c_{q} \\
= & \sum_{p, q} \varphi_{p}(x)^{\dagger} \gamma_{5} \varphi_{q}(x) \frac{1}{Z} \prod_{n}\left(\Omega_{n}+m\right) \frac{\delta_{p, q}}{\left(\Omega_{p}+m\right)} \\
= & \sum_{p} \varphi_{p}(x)^{\dagger} \gamma_{5} \varphi_{p}(x) \frac{1}{\left(\Omega_{p}+m\right)}
\end{aligned}
$$

since

$$
Z=\prod_{n}\left(\Omega_{n}+m\right) .
$$

Consequently,

$$
\begin{aligned}
\lim _{m \rightarrow 0} 2 i m\left\langle\bar{\psi}(x) \gamma_{5} \psi(x)\right\rangle= & 2 i \sum_{\Omega_{k}=0} \varphi_{k}(x)^{\dagger} \gamma_{5} \varphi_{k}(x) \\
= & 2 i\left(\sum_{k=1}^{n_{+}} \varphi_{0, k}^{+}(x)^{\dagger} \varphi_{0, k}^{+}(x)\right. \\
& \left.-\sum_{k=1}^{n_{-}} \varphi_{0, k}^{-}(x)^{\dagger} \varphi_{0, k}^{-}(x)\right),
\end{aligned}
$$

where $n_{+}\left(n_{-}\right)$is the number of positive (negative) chirality zero modes.

Now, with this result one can integrate over space on both sides of Eq. (45) to obtain

$$
\begin{gathered}
\int d^{4} x \sum_{k=1}^{n_{+}} \varphi_{0, k}^{+}(x)^{\dagger} \varphi_{0, k}^{+}(x)-\int d^{4} x \sum_{k=1}^{n_{-}} \varphi_{0, k}^{-}(x)^{\dagger} \varphi_{0, k}^{-}(x) \\
=\frac{1}{32 \pi^{2}} \int d^{4} x \operatorname{tr}\left\{F_{\mu \nu}^{b}(x) F_{\alpha \beta}^{c}(x)\right\} \epsilon^{\mu \nu \alpha \beta},
\end{gathered}
$$

where we have discarded the contribution of the total divergence of $\mathcal{J}_{5}^{\mu}$. Equation (56) can be written in the form

$$
n_{+}-n_{-}=\frac{1}{32 \pi^{2}} \int d^{4} x \operatorname{tr}\left\{F_{\mu \nu}(x) F_{\alpha \beta}(x)\right\} \epsilon^{\mu \nu \alpha \beta},
$$

which is nothing but the index theorem for the Dirac operator $D$.

Then, neither the anomaly (Eq. (45)) nor the index theorem (Eq. (57)) are affected by this minimal Lorentz symmetry violation. Only the expression of the axial current $\mathcal{J}_{5}^{\mu}$ is changed as in Eq. (16).

Let us end this section by noting that an investigation on the relation between Lorentz violation and vector models with a Wess-Zumino term which can be connected with models containing chiral fermions has been reported in [22]. In that case a dynamical Lorentz violation is described as the nonperturbative counterpart of perturbative unitarity breaking in chiral gauge theories due to gauge anomalies.

\section{SUMMARY AND DISCUSSION}

In this work we explored the fate of the chiral anomaly in a fermionic model in which the Lorentz symmetry is explicitly broken by terms which preserve chiral symmetry at the classical level and the form of the Clifford algebra satisfied by the $\Gamma^{\mu}$ matrices replacing Dirac matrices in the Lorentz invariance violating fermionic Lagrangian.

On rotational invariance grounds, only the $c^{0}{ }_{0}$ component was taken as nonvanishing in Eq. (17), so that the energy-momentum relation is changed in the sense that each particle has a maximum attainable velocity (see Eqs. (19) and (20)) which depends only on the dimensionless parameter $\kappa$ (and is independent of the parameter $Q$ ). 
Now, in view of the connection, through the Dirac operator index, between the anomaly and a topological object (the Chern-Pontryagin index) one should expect that the anomaly itself as well as its physical implications (like those related to the $\pi^{0} \rightarrow 2 \gamma$ decay) remain unaltered.

Concerning the anomaly, we have shown, within the path-integral approach and using a heat-kernel regularization, that the Fujikawa Jacobian is not modified. This result was obtained by regularizing the path-integral measure with the same operator that plays the role of the Dirac operator in the classical action, namely, that with a minimal Lorentz violation that classically preserves chiral invariance.

Moreover, the Noether method yields to an anomaly equation for the chiral current that is formally the same as in the Lorentz invariant case, except that the divergence term contains the modified axial current (16). However, since the contribution of such a term vanishes when integrated over all space, the index theorem equation remains unaltered.

We have also discussed within a Lorentz violating framework, the issue of the $\pi^{0} \rightarrow 2 \gamma$ decay, which is connected to the chiral anomaly.

Since the adopted Lorentz symmetry breaking implies different velocities for different massless particles, fermion velocities in the triangle diagram differ from the photon one (Eqs. (20) and (21)). Then, the $\pi^{0}$ decay amplitude in the chiral limit is modified and the change is proportional to $\kappa$ (Eq. (26)), a parameter controlling Lorentz violation. Now, different experimental and phenomenological tests show the smallness of $\kappa$ so that changes in the $\pi^{0} \rightarrow 2 \gamma$ cannot be detected. However if other effects such as the addition of $C P T$ violating terms were considered, the form of the chiral anomaly could be affected and experimental consequences in processes as that of the $\pi^{0}$ decay could become detectable.

\section{ACKNOWLEDGMENTS}

We would like to thank J. Alfaro, M. Asorey, H.O. Girotti, and L. Alvarez-Gaumé for passionate discussions on the subject during the CEFIMAS Buenos Aires Workshop on May 2007. We would like to thank also Prof. W. Bietenholz, Prof. R. Jackiw, and Prof. V. A. Kostelecky for useful comments on this manuscript. This work was partially supported by FONDECYT-Chile and CONICYT grants No. 1050114, No. 1060079, and No. 21050196, PIP6160-CONICET, PIC-CNRS/ CONICET, BID 1728OC/AR PICT20204-ANPCYT grants, and by CIC and UNLP (11/X381 and 11/X450), Argentina.
[1] S. Weinberg, Quantum Field Theory (Cambridge University Press, Cambridge, England, 1995), Vol. I and II.

[2] G. Amelino-Camelia, J. Ellis, N. Mavromatos, D. V. Nanopoulos, and S. Sarkar, Nature (London) 393, 763 (1998); D. Sudarsky, L. Urrutia, and H. Vucetich, Phys. Rev. D 68, 024010 (2003); J. Alfaro, H. Morales-Tecotl, and L. F. Urrutia, Phys. Rev. Lett. 84, 2318 (2000); Phys. Rev. D 65, 103502 (2002); N. R. Bruno, G. AmelinoCamelia, and J. Kowalski-Glikman, Phys. Lett. B 522, 133 (2001); G. Amelino-Camelia, Int. J. Mod. Phys. D 11, 35 (2002); Yi-Fu Cai and Yun-Song Piao, arXiv:gr-qc/ 0701114.

[3] V. A. Kostelecký and S. Samuel, Phys. Rev. D 39, 683 (1989); V. A. Kostelecký Phys. Rev. D 69, 105009 (2004); V. A. Kostelecký and R. Potting, Phys. Rev. D 51, 3923 (1995); D. Colladay and V. A. Kostelecký, Phys. Lett. B 511, 209 (2001); V. A. Kostelecký and R. Lehnert, Phys. Rev. D 63, 065008 (2001); R. Bluhm and V. A. Kostelecký, Phys. Rev. Lett.. 84, 1381 (2000); V. A. Kostelecký and C. D. Lane, Phys. Rev. D 60, 116010 (1999); R. Jackiw and V. A. Kostelecký, Phys. Rev. Lett.. 82, 3572 (1999); D. Colladay and V. A. Kostelecký, Phys. Rev. D 58, 116002 (1998); O. Bertolami, D. Colladay, V. A. Kostelecky, and R. Potting, Phys. Lett. B 395, 178 (1997).

[4] R. Jackiw and S. Y. Pi, Phys. Rev. D 68, 104012 (2003); Z.
Guralnik, R. Jackiw, S. Y. Pi, A. P. Polychronakos, Phys. Lett. B 517, 450 (2001); S. Carroll, R. Jackiw, and G. Field, Phys. Rev. D 41, 1231 (1990); R. Jackiw and S. Y. Pi, Phys. Rev. D 68, 104012 (2003).

[5] J. M. Carmona, J. L. Cortes, J. Gamboa, and F. Mendez, J. High Energy Phys. 03 (2003) 058Phys. Lett. B 565, 222 (2003); A. Das, J. Gamboa, F. Mendez, J. Lopez-Sarrion, J. High Energy Phys. 05 (2004) 022; J. Carmona, J. L. Cortes, A. Das, J. Gamboa, and F. Mendez, Mod. Phys. Lett. A 21, 883 (2006); J. Gamboa and J. Lopez-Sarrion, Phys. Rev. D 71, 067702 (2005); H. Falomir, J. Gamboa, J. Lopez-Sarrion, F. Mendez, and A. J. da Silva Phys. Lett. B 632, 740 (2006); Phys. Rev. D 74, 047701 (2006); J. Gamboa, J. Lopez-Sarrion, and A.P. Polychronakos, Phys. Lett. B 634, 471 (2006); P. Arias, A. Das, J. Gamboa, J. Lopez-Sarrion, and F. Mendez, Phys. Lett. B 650, 401 (2007); A. Das, J. Gamboa, J. Lopez-Sarrion, and F. A. Schaposnik, Phys. Rev. D 72, 107702 (2005); M. Gomes, J. R. Nascimento, E. Passos, A. Yu. Petrov, and A. J. da Silva, arXiv:0704.1104; A. F. Ferrari, M. Gomes, J. R. Nascimento, E. Passos, A. Yu. Petrov, A. J. da Silva, arXiv:hep-th/0609222.

[6] A. A. Andrianov, P. Giacconi, and R. Soldati, J. High Energy Phys. 02 (2002) 030; J. Alfaro, A. A. Andrianov, M. Cambiaso, P. Giacconi, and R. Soldati, Phys. Lett. B 639, 586 (2006). 
[7] O. Bertolami and C. S. Carvalho, Phys. Rev. D 61, 103002 (2000).

[8] V. A. Kostelecký and M. Mewes, Phys. Rev. D 69, 016005 (2004).

[9] V. A. Kostelecký and M. Mewes, Phys. Rev. D 66, 056005 (2002).

[10] S. Coleman and S. L. Glashow, Phys. Lett. B 405, 249 (1997).

[11] S. Coleman and S. L. Glashow, Phys. Rev. D 59, 116008 (1999).

[12] M. C. Gonzalez-Garcia and M. Maltoni, arXiv:0704.1800.

[13] S. L. Adler, Phys. Rev. 177, 2426 (1969).

[14] J. S. Bell and R. Jackiw, Nuovo Cimento A 60, 47 (1969).

[15] S. K. Lamoreaux, J. P. Jacobs, B. R. Heckel, F. J. Raab, and E. N. Fortson, Phys. Rev. Lett. 57, 3125 (1986); B. Altschul, Phys. Rev. D 75, 041301 (2007); Phys. Rev. Lett. 96, 201101 (2006).

[16] P. Wolf, F. Chapelet, S. Bize, and A. Clairon, Phys. Rev.
Lett. 96, 060801 (2006)

[17] P. L. Stanwix, M. E. Tobar, P. Wolf, C. R. Locke, and E. N. Ivanov, Phys. Rev. D 74, 081101 (2006); S. Herrmann, A. Senger, E. Kovalchuk, H. Müller, and A. Peters, Phys. Rev. Lett. 95, 150401 (2005); P. Antonini, M. Okhapkin, E. Goeklue, and S. Schiller, Phys. Rev. A 71, 050101 (2005); J. A. Lipa, J. A. Nissen, S. Wang, D. A. Stricker, and D. Avaloff, Phys. Rev. Lett. 90, 060403 (2003).

[18] L. B. Auerbach et al., Phys. Rev. D 72, 076004 (2005); T. Katori et al., Phys. Rev. D 74, 105009 (2006).

[19] R. E. Gamboa Saravi, M. A. Muschietti, and J. E. Solomin, Commun. Math. Phys. 93, 407 (1984).

[20] K. Fujikawa, Phys. Rev. Lett. 42, 1195 (1979); Phys. Rev. D 21, 2848 (1980).

[21] R.E. Gamboa Saraví, M.E. Muschietti, F. Schaposnik, and J. Solomin, Ann. Phys. (N.Y.) 157, 360 (1984).

[22] A. A. Andrianov and R. Soldati, Phys. Rev. D 51, 5961 (1995). 